Katarzyna Znajdek*, Natalia Szczecińska, Maciej Sibiński, Przemysław Czarnecki, Gabriela Wiosna-Satyga, Aleksandra Apostoluk, Fabien Mandrolo, Szymon Rogowski, and Zbigniew Lisik

\title{
Energy converting layers for thin-film flexible photovoltaic structures
}

https://doi.org/10.1515/phys-2018-0102

Received September 21, 2017; accepted October 27, 2018

\begin{abstract}
The paper presents research focused on the efficiency improvement of inorganic flexible thin-film solar cells, using energy converting layers. The light capture enhancement was achieved through the introduction of layers based on rare-earth elements, as top coatings on the amorphous silicon photovoltaic structures. Such luminescent layers are converting high-energy photons into low-energy ones, which are more efficient in photovoltaic conversion of the investigated solar cells. Towards this goal, powders consisting rare-earth elements were applied as active particles in polymer layer. For practical experiments, the screen-printing method, as a cheap, reliable and industrially-ready technology was used for layers deposition. For the experiments two compositions were selected: Sr4Al14O25: Eu,Dy (BGL-300M) and SrAl2O4: Eu,Dy (G-300M). These materials are characterized by excellent thermal and optical stability and interesting luminescent properties (they absorb ultraviolet and emit in the visible range). For the verification of investigated materials and methods, various compositions of powders and proportions were tested and analyzed.
\end{abstract}

Keywords: energy down-conversion, thin-film solar cells, flexible photovoltaic structures, rare-earth elements.

PACS: 47.54.Jk, 88.40.hj, 88.40.jj, 81.07.Wx, 82.35.Np

\footnotetext{
*Corresponding Author: Katarzyna Znajdek: Łódź University of Technology, Żeromskiego 116, 90-924 Łódź, Poland, E-mail: katarzyna.znajdek@p.lodz.pl

Natalia Szczecińska: Łódź University of Technology, Żeromskiego 116, 90-924 Łódź, Poland,

E-mail: nataliaszczecinska@tlen.pl

Maciej Sibiński: Łódź University of Technology, Żeromskiego 116, 90-924 Łódź, Poland, E-mail: maciej.sibinski@p.lodz.pl Przemysław Czarnecki: Łódź University of Technology, Żeromskiego 116, 90-924 Łódź, Poland, E-mail: 201937@edu.p.lodz.pl Gabriela Wiosna-Sałyga: Łódź University of Technology, Żeromskiego 116, 90-924 Łódź, Poland, E-mail: gabriela.wiosna-salyga@p.lodz.pl
}

\section{Introduction}

Permanent growth of the world global energy needs requires novel technological solutions that would meet this increasing demand. Simultaneously, the environment protection policy accompanied by the concept of energy sources diversification, leads to growing interest in the increase of renewable energy usage. Photovoltaic conversion is considered as one of the main roads successfully fulfilling the growing energy demand.

The development of photovoltaics in recent years has been concentrated on activities aimed at reducing the costs of photovoltaic (PV) devices manufacturing, as well as at the improvement of their photovoltaic conversion efficiency. Adjusting both of these factors at the suitable levels could make photovoltaics competitive with the conventional energy sources. Introducing innovative solutions, such as thin-film and flexible photovoltaic structures, substantially broadens the possibilities of the implementation of solar cells.

The objective of the presented research aims at novel solar cell structures with enhanced efficiency, due to the introduction of energy converters. Authors propose the application of thin films composed of rare-earth (RE) elements, or their mixtures with $\mathrm{ZnO}$ nanoparticles (NPs), as luminescent down-converting layers. The idea is to convert high-energy photons (UV light) into lower energy ones (visible light) which are more efficient in the photovoltaic conversion. General concept of the down-conversion and down-shifting process using nanoparticles is shown in Fig-

\footnotetext{
Aleksandra Apostoluk: Université de Lyon and INL, CNRS, UMR 5270, INSA Lyon, France,

E-mail: aleksandra.apostoluk@insa-lyon.fr

Fabien Mandrolo: Université de Lyon and INL, CNRS, UMR 5270, INSA Lyon, France, Email: fabien.mandorlo@insa-lyon.fr Szymon Rogowski: Łódź University of Technology, Żeromskiego 116, 90-924 Łódź, Poland, Poland, E-mail: 801180@edu.p.lodz.pl Zbigniew Lisik: Łódź University of Technology, Żeromskiego 116, 90-924 Łódź, Poland, E-mail: zbigniew.lisik@p.lodz.pl
} 


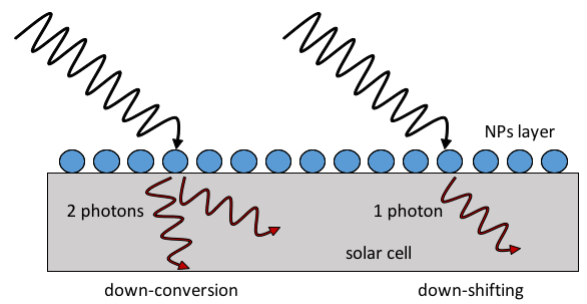

Figure 1: Schematic illustration of the energy down-conversion and down-shifting concept, using nanoparticles as front layer of a solar cell, based on Ref. [2]. The losses by reflection at the interface of the air and the energy converter layer are not shown.

ure 1. A down-converting layer placed on the front side of a solar cell has the potential to generate more than one low-energy photon (precisely speaking, it generates two photons) per every incident high-energy photon. Many decades of research have been dedicated to the optimization of photovoltaic devices and it is anticipated that further advances can be made by modifying the input spectrum rather than the electronic properties of the PV structure [1].

In practice, a lot of research on luminescence downconversion with an external quantum efficiency (EQE) of less than unity has been performed for shifting the shorter wavelengths to longer ones [1]. The application of down converting layers in PV includes luminescent solar concentrators [3-5] and measures to overcome limitations in the front surface of some solar cell designs [6]. For example, the luminescent silicon nanoparticle/polymer composite films that provided UV wavelength to visible light down-conversion, were proposed [7]. Other methods for light trapping, in order to increase the absorption in the solar cell active material, and hence boost cell's efficiency, use the phenomenon of localized surface plasmon excitation in metallic nanoparticles, which are deposited on the semiconductor surface. There were already some theoretical and practical investigations performed in this area, with the utilization of silver nanoparticles for enhancing the absorbance of silicon solar cells [8,9]. It was shown that silver nanoparticles deposited onto the surface of a single p-n junction silicon solar cell can slightly enhance the light absorption and hence increase the photocurrent generation in the active silicon layer [9]. There is also some research made on rare-earth-doped luminescent materials in the application as down-converting layers to enhance solar cell performance [5]. In our previous work, we proposed the application of zinc oxide nanoparticles as a down-converting layer $[10,11]$. The preliminary results led us to the new proposal, which combines two last meth-



Figure 2: AM 1.5 spectrum (dashed curve) and the external quantum efficiency (EQE) of the amorphous silicon (a-Si) solar cells, showing the mismatch between the solar illumination spectrum and the efficiency curve. Clear pink and dark pink rectangles represent, respectively, the UV spectral zone which is turned the visible light thanks to the down-conversion or down-shifting phenomenon.

ods and includes an easy-to-implement deposition technology.

\section{Approach and methods}

The results presented in this paper focus on the efficiency improvement of thin-film flexible solar cells made of amorphous silicon. As it is shown in Figure 2, there is a high potential for the down-conversion in that type of PV structures. External quantum efficiency for these solar cells is very low in the shorter wavelengths range (presented in clear pink in Figure 2). The proposed approach is to use rare-earth elements and $\mathrm{ZnO}$ nanoparticles to obtain down-conversion or down-shifting phenomenon (in the wavelength range presented in dark pink in Figure 2).

Rare-earth elements are chosen because of their high luminescent quantum efficiency. Another big advantage of this kind of materials is that they are usually characterized by the phenomenon of phosphorescence, hence it is possible not only to use the emission of elements during the irradiation, but also the emission after the exposure. Unfortunately, these elements have narrow emission and excitation spectra, therefore we fabricated some mixtures including these elements with various compositions, in order to improve their properties.

In the first series of experiments, two types of RE elements' components have been chosen basing on the previous investigations. Materials called BGL-300M (Sr4Al14O25: $\mathrm{Eu}$, Dy) and G-300M (SrAl2O4: Eu, Dy) were used. They are characterized by the excellent thermal and optical stability and interesting luminescent properties [12]. In the ex- 
perimental process, RE powders were first milled using a ball mill equipment in order to create smaller particles. The results of milling for BGL-300M material are shown in Figure 3. In the next step, the luminescent properties of such prepared powders have been measured and analysed. Subsequently, in order to prepare optimized compositions for screen-printing deposition, a proper polymer matrix has been selected, in which milled RE powders were dispersed. After the layers' deposition onto aSi flexible thin-film solar cells, EQE measurements have been conducted. For the further energy conversion improvement, investigated RE compositions were mixed with two types of ZnO NPs powders, with nanoparticles' diameters of $20 \mathrm{~nm}$ and from 90 to $210 \mathrm{~nm}$. The results demonstrate that both types of materials (RE elements in a polymer matrix only or RE elements mixed with ZnO NPs in a matrix) have very promising optical properties.

\section{Results}

For all prepared samples, including BGL-300M and G$300 \mathrm{M}$ powders, as well as two types of $\mathrm{ZnO}$ nanoparticle powders (20 nm and 90-210 nm, respectively) and their compositions, optical measurements were conducted in order to check their suitability as photon converting layers for photovoltaic applications. Excitation and emission spectra for all investigated samples were measured using fluorescence spectroscopy with FLS920 computer controlled spectro-fluorimeter (Edinburgh Instruments), the equipment for measuring steady state luminescence spectra in the ultraviolet to near infrared spectral range, with single photon counting sensitivity. Microscopic images were taken using Keyence VHX optical 3D microscope.

The initial sets of experiments were conducted on the chosen compositions of rare-earth elements' mixtures. Selected powders (BGL-300M and G-300M) were first crumbled using ball mill equipment. The aim of this treatment was to obtain smaller particles for better down-conversion efficiency. Although the decrease of particles size is ob-



Figure 3: Microscope images of BGL-300M mixture powder, from the left: not milled, milled for 10 and 20 minutes.

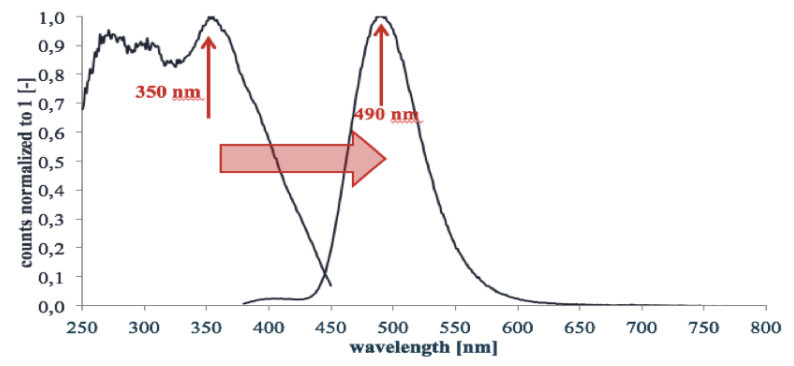

Figure 4: The excitation (left) and emission (right) spectrum of Sr4Al14025: Eu,Dy (BGL-300M).



Figure 5: The excitation (left) and emission (right) spectrum of SrAl204:Eu,Dy (G-300M) sample.

served (Figure 3), there is no significant difference in the energy-conversion performances of milled and not milled powder of investigated REs. Figures 4 and 5 show the excitation and emission spectra of both studied materials (BGL-300M and G-300M).

The wide part of the Sr4Al14O25: Eu,Dy component's excitation spectrum is situated in the part of UV spectral zone. It can be observed that investigated RE powder converts this radiation to the visible light. The maximum emission of this sample is at $490 \mathrm{~nm}$.

Very similar situation occurs in the case of SrAl2O4:Eu,Dy, the second studied material. The maximum excitation for this one is situated between $370-400$ $\mathrm{nm}$. The powder absorbs the UV radiation and converts it into the visible light. The maximum emission is observed at $520 \mathrm{~nm}$. Comparing maximum emission values for both cases with the Figure 2, it can be concluded that the emission ranges for both BGL-300M and G-300M fits the external quantum efficiency characteristics of a-Si solar cell.

In order to deposit down-converting layers on flexible thin-film solar cells, it was necessary to prepare a dispersion of selected RE mixtures. BGL-300M and G-300M were dispersed, in the solution containing PMMA (poly(methyl methacrylate)) and carbitol butyl acetate. Layers were deposited using screen-printing technology, which is well 




Figure 6: Optical microscope images and surface profiles of Sr4Al14025: Eu,Dy (BGL-300M) layers.

known, one of the cheapest, and can be easily implemented in the industry. Figure 6 presents optical microscope images of a such deposited layer containing 30\% (in weight) BGL-300M, together with its 3D surface profile.

Crucial tests in the evaluation of the suitability of the proposed materials as down converting layers for the efficiency improvement of a-Si solar cells, are the external quantum efficiency (EQE) measurements. For comparison, reference solar cell was used with no additional layer (referred as "none" in the Figure 7). Dispersions containing $10 \%$ and $30 \%$ (in weight) of the studied RE materials were tested. Results of EQE measurements on the a-Si solar cells with the deposited down-converting layers on their top are presented in Figure 7.

Authors investigated three levels of RE content, namely $10 \%, 30 \%$ and $50 \%$. Specific investigation results of the optical transmittance measurements, presented in [13], proved that the $50 \%$ level of RE admixture significantly reduced the visible light transmission efficiency, and thus appeared negative for the overall cell performance. Considering $10 \%$ content of tested admixtures (especially in BGL case) the luminescence effectiveness was insufficient for practical application, which is depicted in Figure 7.

It can be observed that the first investigated composition (BGL-300M) has more significant influence on the EQE characteristics of a-Si photovoltaic structures. Comparing to the reference solar cell without any down-converting layer, there is a substantial increase of EQE values in the UV spectral zone, especially for the concentration of 30\% of RE materials. The second RE composition (G-300M) appeared not suitable for the proposed application. For the higher concentration of $30 \%$ there is only a very weak in-

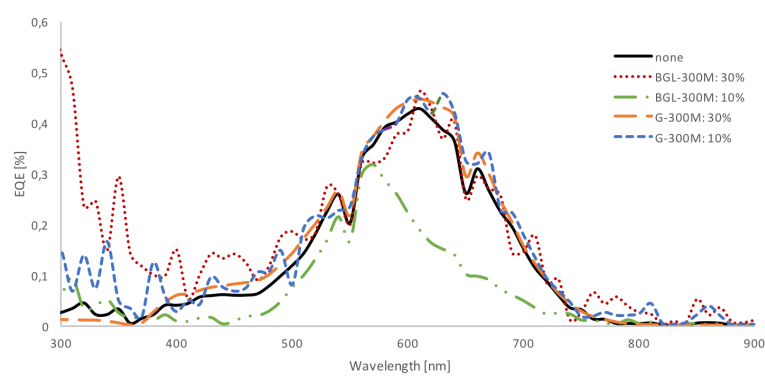

Figure 7: External quantum efficiency results for flexible a-Si solar cells with and without down-converting layers based on BGL-300M and G-300M rare-earth compositions, with various concentrations in the initial dispersion.

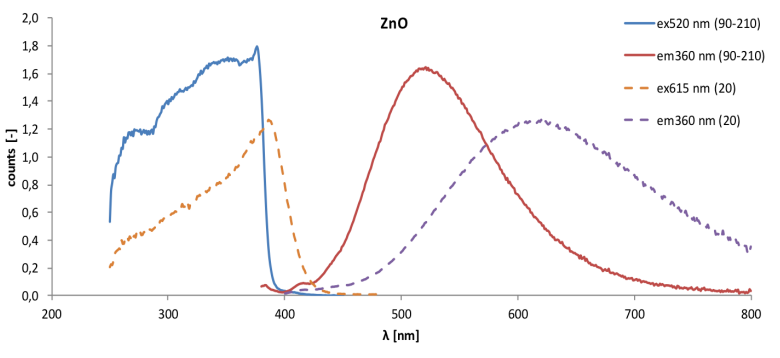

Figure 8: The excitation and emission spectra of $\mathrm{ZnO}$ nanopowders with different nanoparticle sizes ( $20 \mathrm{~nm}$ and 90-210 nm, respectively). The excitation wavelength for each curve is indicated in the legend.

fluence on the EQE of the solar cell in the UV, while for lower concentration of $10 \%$ the influence is detrimental.

The objective of our further research was to broaden the emission spectrum of the investigated down converting layers. Thus, basing of the previous experience with $\mathrm{ZnO}$ nanoparticles, we propose to prepare mixtures of the studied RE elements compositions with two types of $\mathrm{ZnO}$ nanoparticle powders - with the sizes of 20 and $90-210 \mathrm{~nm}$ in NPs diameter. Excitation and emission spectra of both pure $\mathrm{ZnO}$ NP powders are shown in Figure 8. The emission range in both cases is much wider than for the investigated RE element powders alone. Thus, the improvement of the performances of the RE elements- $\mathrm{ZnO}$ mixtures is expected.

All samples of ZnO-RE mixtures were prepared using ball milling in with two different milling times: 10 and 20 minutes. All samples were prepared in 1:1 mass proportions. Figures 9 and 10 show the excitation and emission spectra of the material containing RE elements mixed with $20 \mathrm{~nm} \mathrm{ZnO} \mathrm{NPs,} \mathrm{while} \mathrm{Figures} 11$ and 12 present the curves obtained for RE mixtures with ZnO NPs of 90-210 $\mathrm{nm}$. All spectra were measured for the studied materials in 


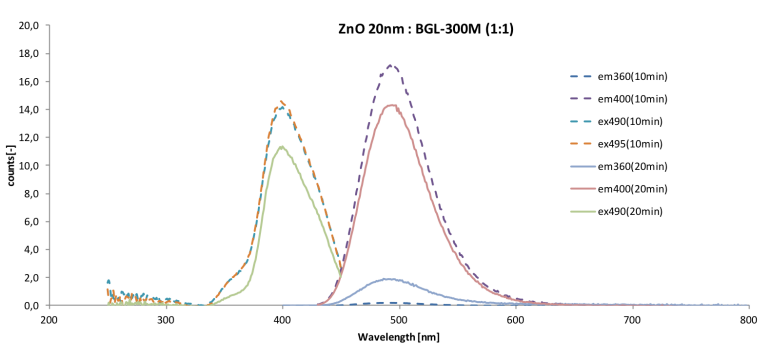

Figure 9: The excitation and emission spectra of mixtures BGL$300 \mathrm{M}-\mathrm{ZnO}$ (NPs of $20 \mathrm{~nm}$ ), milled for 10 and 20 minutes. The excitation wavelength for each curve is indicated in the legend.

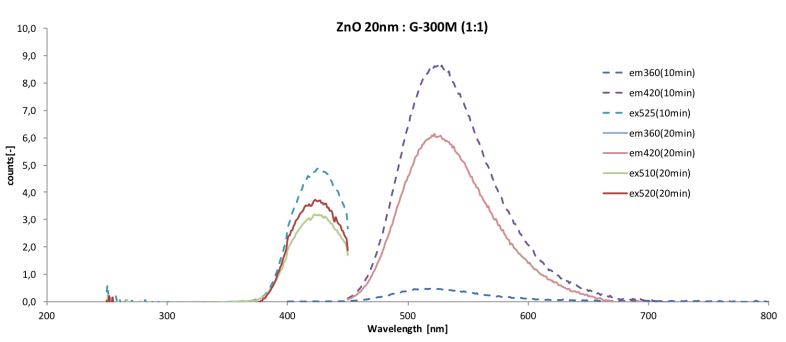

Figure 10: The excitation and emission spectra of mixtures G-300M$\mathrm{ZnO}$ (NPs of $20 \mathrm{~nm}$ ), milled for 10 and 20 minutes. The excitation wavelength for each curve is indicated in the legend.

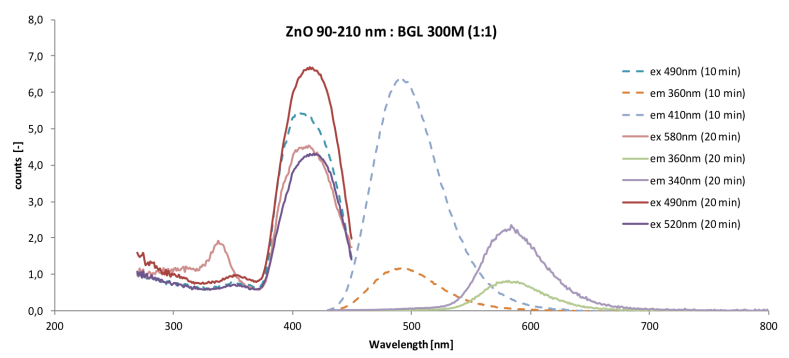

Figure 11: The excitation and emission spectra of mixtures BGL$300 \mathrm{M}-\mathrm{ZnO}$ (NPs of $90-210 \mathrm{~nm}$ ), milled for 10 and 20 minutes. The excitation wavelength for each curve is indicated in the legend.

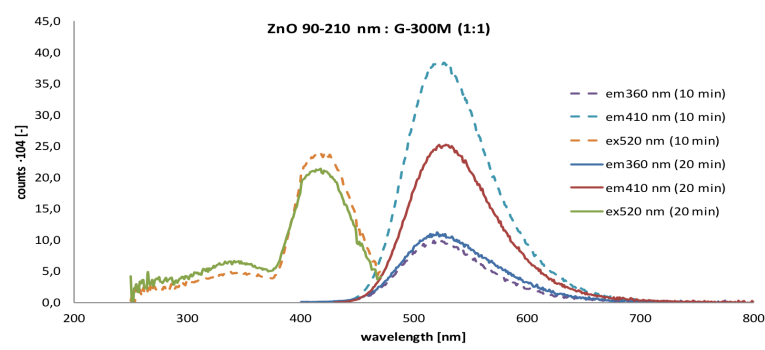

Figure 12: The excitation and emission spectra of mixtures G-300M$\mathrm{ZnO}$ (NPs of $90-210 \mathrm{~nm}$ )), milled for 10 and 20 minutes. The excitation wavelength for each curve is indicated in the legend. the form of the powder (before preparing the final downconverting layers).

The obtained results show that the emission in visible range for both compositions (BGL-300M-ZnO (NPs of $20 \mathrm{~nm}$ ) and G-300M-ZnO (NPs of $20 \mathrm{~nm}$ )) in the region of 500-530 nm. Higher values of the emission were observed for the first composition. Thus the results are promising for the proposed application of these materials as the energy converters in thin-film flexible photovoltaic structures.

The results for the mixtures of RE elements and $\mathrm{ZnO}$ of bigger nanoparticle size $(90-210 \mathrm{~nm})$ also reveal the absorption in UV and the emission in the visible range. Analogically, as for previous set of samples (RE elements mixed with ZnO NPs of $20 \mathrm{~nm}$ in size), maximum emission is observed at about $500 \mathrm{~nm}$ for the mixture of BGL-300MZnO NPs of 90-210 nm in size, and at about $525 \mathrm{~nm}$ for G$300 \mathrm{M}-Z n O$ NPs of $90-210 \mathrm{~nm}$ in size mixture.

Unfortunately, the spectral range of the emission does not change significantly for both mixtures of RE elements and $\mathrm{ZnO}$ of either $20 \mathrm{~nm}$ or $90-210 \mathrm{~nm}$ in diameter, when compared to the RE element powders alone. Following this observation, the preparation of the dispersion containing ZnO NPs mixed with RE elements and the deposition of the layers on solar cells was abandoned.

\section{Conclusions}

In the presented paper, the luminescent and optical properties of $\mathrm{RE}$ elements compositions and $\mathrm{ZnO}$ powders were studied. The screen-printing method was used as an effective, relatively uncomplicated and easily scalable layer deposition technique. As a result, a series of homogeneous layers on thin-film flexible solar cells was obtained and investigated. External quantum efficiency curves showed that there is an increase in the solar cell efficiency value in the low wavelengths region after deposition of the downconverting layer containing BGL-300M material.

The excitation and emission spectra of the investigated rare-earth element compositions, $\mathrm{ZnO}$ nanoparticles as well as their mixtures show that these materials can be effectively used for energy shifting of the UV into visible in order to improve the EQE characteristic of a-Si solar cell. However, the decrease of the rare earth elements grain size in the grinding process does not influence their luminescent properties significantly.

It was also proven that examined materials can be successfully incorporated into a polymer matrix in order to obtain an efficient and mechanically stable luminescent layer to be deposited on a flexible thin-film a-Si solar 
cell. This shows that it is possible to use proposed RE elements as a down-converting material in order to improve the properties of thin-film flexible photovoltaic cells.

Acknowledgement: Presented work was supported by the National Science Centre, Poland, within the research project SONATA 11 number: 2016/21/D/ST7/01215.

\section{References}

[1] Richards B.S., Luminescent layers for enhanced silicon solar cell performance: Down-conversion, Sol. Energy Mater. Sol. Cells, 2006, 90 (9), 1189-1207.

[2] Abrams Z. R., Niv A., Zhang X., Solar energy enhancement using down-converting particles: A rigorous approach, J. Appl. Phys., 2011, 109, 114905.

[3] Goetzberger A., Gruebel W., Solar Energy Conversion with Fluorescent Collectors, Appl. Phys., 1977, 14 (2), 123-139.

[4] Friedman P. S., Parent C. R., SERI subcontract no. XE-2-02145-01, 1987, 193.

[5] Richards B.S., Shalav A., Corkish R.P., A low escape-cone loss luminescent solar concentrator, 19th Europ. Conf. Photovolt. Solar Ener. Conv., Paris, 2004, 113-116.

[6] Maruyama T., Enomoto A., Shirasawa K., Solar cell module colored with fluorescent plate, Sol. Energy Mater. Sol. Cells, 2000, 64, 269-278.

[7] Nayfeh M.H., United States Patent No: US 2009/0102353 A1
[8] Akimov Y.A., Ostrikov K., Li E.P., Surface Plasmon Enhance ment of Optical Absorption in Thin-Film Silicon Solar Cells, Plasmonics 4, 2009, 107-113.

[9] Pillai S., Catchpole K.R., Trupke T., Green M.A., Surface plasmon enhanced silicon solar cells, J. Appl. Phys., 2007, 101, 093105.

[10] Apostoluk A., Zhu Y., Masenelli B., Delaunay J.-J., Sibinski M., Znajdek K., Focsa A., Kaliszewska I. , Improvement of the solar cell efficiency by the $\mathrm{ZnO}$ nanoparticle layer via the downshifting effect, Microelectronic Engineering, 2014, 127, 51-56.

[11] Znajdek K., Sibinski M., Lisik Z., Apostoluk A., Zhu Y.,Masenelli B., Zinc oxide nanoparticles for improvement of thin film photovoltaic structures efficiency through down shifting conversion, Opto-Electronics Review, 2017, 25, 99-102.

[12] Nemoto Lumi-materials CO., LTD., Phosphorescent Pigments Characterization, https://nemoto.co.jp, 20.07.2017

[13] Znajdek K., Szczecińska N., Sibiński M., Wiosna-Sałyga G., Przymecki K., Luminescent layers based on rare earth elements for thin-film flexible solar cells applications, Optik, 2018, 165, 200-209.

[14] Almosni S. et al., Material challenges for solar cells in the twenty-first century: directions in emerging technologies, Sci. Techn. Adv. Mat., 2018, 19 (1), 336-360.

[15] Wang H., Jiang S., Xiang G., Li L., Tang X., Luo X., Zhou X., Preparation, structure and down-shifting luminescence of $\mathrm{Yb} 3+$ doped KLa505(VO4)2, Mat. Res. Bulletin, 2018, 108, 5-9.

[16] Zhao R. et. al., Double-shell CeO2:Yb, Er@SiO2@Ag upconversion composite nanofibers as an assistant layer enhanced nearinfrared harvesting for dye-sensitized solar cells, J. All. Comp., 2018, 769, 92-95. 